\title{
The Elimination Strategy Of We-media Image
}

\author{
Zhang Jianxiong1,a, , Fu Yinglin ${ }^{2, b}$ Youqiang $\mathrm{We}^{3, \mathrm{c}}$ \\ 1,2,3School of Journalism \& Communication, Jiangxi Normal University, Nanchang, Jangxi, China \\ a853044324@qq.com, b1341249040@qq.com, c190555223@qq.com \\ "Zhang Jianxiong
}

Keywords: We-media,Spread the image,Negative public opinion,Elimination strategy

\begin{abstract}
With the continuous development of communication technology and the enhancement of people's consciousness of independent communication, the self media has been developing rapidly at present, and it is disseminated independently through different communication channels in different media platforms. More and more people are pouring into the media and participating in it, actively acting as the disseminators and information makers of information, adding to the low threshold of communication from the media, the relative freedom of speech, the quality of the media users, and so on, with the expansion of the media platform and the mass growth of the media users. China's self media management system has not kept pace with the speed of development, and the problems existing in our media have been increasingly exposed to the public, especially the fierce competition from the media. Many media disseminators, such as heading party, washing manuscript and fabricating facts, have appeared in order to achieve the purpose of increasing powder and attracting attention. The ball, from the media also often exaggerates the fact, in the process of the spread of negative news, the media often play a role in boosting the flames, and the government if the negative public opinion spread and formed by the self media close to the audience is not handled properly, it will have an extremely bad influence on social public opinion.
\end{abstract}

\author{
自媒体传播乱像的消解策略 \\ 张建熊 $1, \mathrm{a}$, 傅应林 $2, \mathrm{~b}$, 魏友强 $3, \mathrm{c}$ \\ $1,2,3$ 江西师范大学新闻与传播学院, 南昌, 江西, 中国 \\ a853044324@qq.com, b1341249040@qq.com, c190555223@qq.com \\ *张建熊
}

关键词: 自媒体; 传播乱像; 负面與情; 消解策略

中文摘要. 随着传播技术的不断发展与人们自主传播意识的增强, 自媒体在当前得到迅速的发 展, 并在不同的自媒体平台通过不同的传播渠道进行自主传播。越来越多的人涌入到自媒体 并参与其中, 主动地充当信息的传播者与信息的制造者, 再加上自媒体有传播门槛较低, 言 论发表相对自由, 自媒体用户素质良莠不齐等特征, 伴随自媒体平台扩张、自媒体用户大量 增长等因素, 我国的自媒体管理制度没有跟上发展的速度, 我国自媒体存在的问题也日益暴 露在公众眼里, 特别是当前自媒体竞争激烈, 许多自媒体传播者为了达到涨粉、吸引关注等 目的, 出现诸如标题党、洗稿、捏造事实等问题, 为了博眼球, 自媒体也常常夸大事实, 在 负面消息的传播过程中, 自媒体常常起到推波助澜的作用, 而政府如果对于贴近受众的自媒 体所传播并形成的负面舆情处理不当，就会对社会與情产生极其恶劣的影响。 


\section{1. 引言}

随着自媒体逐渐贴近群众生活, 并在大众娱乐上担当重要角色, 自媒体传播的优势在不 断地凸显出来，但在自媒体具有传播主体平民化、传播用语标签化、传播内容个性化、传播 效果放大化等特点的同时, 再结合当前自媒体主动拉取和创造信息, 传播内容先进入大众视 野再过滤, 自媒体用户素质良莠不齐, 失实传播失控以及权威部门缺位等不足, 自媒体传播 乱像已经充斥在受众的周围, 并对社会言论起到不良的影响, 特别是在负面新闻的传播中起 到重要的推动作用, 并往往形成负面與情, 对社会秩序和社会言论造成极大的负面伤害, 甚 至有些自媒体传播者已经构成犯罪而不自知, 当前减少自媒体传播乱像, 消解自媒体传播的 负面影响, 并营造一个良好的社会言论氛围是当下值得思考并讨论的问题之一, 下面是我的 建议:

\section{2. 严控自媒体准入标准, 剔除不良自媒体}

随着自媒体的平台如微博、微信等日渐增多, 自媒体的准入门槛已经近乎没有, 随便一 个人都能通过自媒体平台将自己的言论发布到公众平台, 让所有人看到。自媒体的准入没有 门槛, 带来的自然是自媒体的进入者素质也有高有低, 而低素质的自媒体传播者为了达到传 播效果, 会在传播内容上大做文章, 但往往自媒体传播者消息的来源, 事实的依据以及相关 有力的事实证明并不充分, 一味迎合市场, 置内容传播相关方利益于不顾, 甚至置国家和人 民的利益于不顾，给社会與论带来不利影响，造成负面與情。

自媒体准入门槛过低, 自媒体平台要严格把控入口, 严查封闭出口, 对于一些发布不实 信息, 抄袭, 造谣生事并给社会带来巨大不良影响的自媒体用户, 予以查封账号, 并在一段 时间内不允许进入到自媒体平台, 对于情节相当严重者, 甚至要封杀其自媒体账户, 并以造 谣等相关违反法律法规的行为予以法律制裁, 永远禁止其在自媒体平台的传播行为; 对于自 媒体发布的内容, 相关自媒体平台要加强审核, 也要鼓励平台参与者对自媒体的不良行为积 极进行举报, 在接到举报后, 平台要及时进行再次审查, 检验内容是否真实可靠, 对不符合 事实的内容及时进行删除, 并对自媒体传播者进行警告, 造成重大影响者, 可向政府相关部 门进行举报, 追究责任。

\section{3. 权威部门加强监控, 严厉打击自媒体不法行为}

自媒体准入的低门槛, 不仅带来自媒体用户的大量增长, 还带来了一系列之前传统媒体 不存在的问题, 如洗稿侵权, 捏造事实, 出现谣言、虚假信息, 内容低俗等, 而自媒体也不 像传统媒体具有严格的意义上的 “守门人”，对于发布的内容有审核把关。且由于网络具有一 定的隐蔽性和难以追责性, 再加上我国造谣、传谣等的处罚力度较低, 谣言的源头很难查询 等, 使这类犯罪成本较低，也使很多自媒体传播者有恃无恐，多次触及传播底线。

面对这些问题，相关部门要完善立法和追责制度，加大对自媒体违法犯罪行为的处罚力 度, 对待那些造谣、传谣, 并造成恶劣社会影响的自媒体, 坚决予以处罚或是永久禁止准入。 除了加强制度管理和处罚力度, 相关部门也要时刻注意舆情风向, 特别是对待正处在发酵期 的负面與情, 要有敏锐的嗅觉, 在相关负面與情还未在大范围广泛传播的时候及时进行干预, 对不符合事实并有造谣行为的自媒体传播者, 追究其法律责任, 对于严重者, 可予以罚款及 刑事拘留; 相关部门也要加强与自媒体传播平台合作, 加强监督与指导, 时刻注意舆论发展 动态, 对于一些可能引爆與论的话题要及时进行正确的处理。

\section{4. 完善信息公开制度，拦截自媒体失实传播}

自媒体传播者善于追踪热点和引爆舆论, 有时会为了增加热度, 吸引流量, 而枉顾事实, 
夸大事实, 以达到传播的目的。自媒体运营者有时为了急于求成, 在内容和形式上会大胆创 新, 但也因为过于追求标新立异, 而不注重实际内容, 并对有些事件过度解读, 而造成一些 本应该是正面或是不应该有很大传播影响的负面内容大肆传播, 对社会言论及风气造成不良 的影响，从而造成负面舆情。

面对自媒体炒作不实言论, 相关方除了要及时监控、拦截之外, 为了不让不实言论再以 负面的形式传播并扩大影响, 相关方也要及时对自媒体传播的内容进行取证, 做到信息公开 透明, 让受众了解事件的经过和真相, 以防止被自媒体不断的炒作与传播, 如果在事件真相 与事实清楚的情况下, 还有自媒体在进行不良传播, 则要依法追究其法律责任, 绝对不能给 自媒体传播不实信息的机会，并在事件持续发酵前及时阻断不良传播的可能性，给自媒体， 也给受众一个交代; 不应为了防止不良影响扩大, 采取简单粗暴的直接删稿、或是封号的手 段, 这不仅会让自媒体和受众情绪扩大化, 也容易激化矛盾, 使事件的传播与影响走向更加 难以控制的相反面。除了相关部门要取证自媒体的传播内容, 相关自媒体平台也需要审查自 媒体所传播的事实源头, 对于自媒体的不实内容, 要及时澄清, 并予以警告, 并及时发布相 关自媒体警告或是处罚信息, 积极配合国家相关部门对于自媒体信息的取证, 完善自媒体的 实名登记制度，保障信息真实可靠。

\section{5. 净化网络环境，提高网民素质}

在网络环境里, 网民素质良莠不齐的现象一直是造成自媒体不良传播的重要原因之一, 也正因为网络世界里具有 “隐身” “匿名” 等难以监控的死角, 这给网络的不实传播带来了审 查的难题, 而有些低素质网民也正借着这种不易察觉的形式, 在网上大肆造谣、传播谣言, 并利用自媒体进行炒作, 而往往自媒体对来自网络上的信息也是人云亦云, 并不会去核查事 件的真相和事实, 且自媒体不像传统媒体, 讲究客观性, 更多的是带有自己的主观意向, 传 播的内容在很大程度上也是根据自己的喜好和兴趣, 对于某件事情的评价也是站在自己的立 场上, 对事情的传播往往不够客观, 而非专业的自媒体传播者也缺乏对事件客观传播的理论 与实践基础。

净化网络环境, 提高网民素质不是一朝一夕的事情, 也不是某个人或是某个部门的事情。 作为网络时代最具有发言权的网民和自媒体用户, 既要自觉地准守网络和现实中的规则, 也 要为网络环境的优化贡献自己的力量, 面对网络上的不实言论, 要敢于积极主动地站出来谴 责、举报。政府部门也要积极倡导良好的上网行为, 尽可能的保障绿色的网络环境, 积极鼓 励自媒体用户对造谣、传谣等违法行为进行举报, 并对自媒体造成和产生的后果及时跟进, 并及时追究责任, 对待积极向上, 具有正能量的自媒体传播者要进行推广和宣传, 形成榜样 和标杆，推动具有正确价值观的自媒体发展壮大。

\section{6. 发挥正面引导机制, 消除不良影响}

自媒体时代，一个很小的细节、一个很小的过错都会被放大，然后被无限传播，特别是 对于一些负面的、消极的消息来说，被传播和夸大的可能性要远远大于正面和积极的消息。 这些被恶意夸大并被肆意传播的带有一定虚假性的消息, 会在传播过程中不断地被添枝加叶, 并在不同自媒体之间不断地进行错误解读, 并衍生出许多虚假恶意的消息, 以至于社会的阴 暗面被无限放大, 而掩盖了其中真而美的存在, 这反而让一些虚假的东西遮住了受众的眼睛, 在很大程度上影响了社会的风气并造成难以消除的影响。

在自媒体时代, 相关权威部门一定要利用自身的权威性, 对于自媒体发布的相关不实消 息要进行及时的辟谣, 并对传播、影响巨大的消息进行相关积极正面的引导, 对于社会中以 及自媒体传播的正能量的人物、事件要进行推广与传播, 加强与自媒体大号的联系, 借助自 媒体大号的传播影响, 积极进行正面传播, 这在借助自媒体传播优势的同时, 也让自媒体逐 
渐向正面以及规范靠拢, 也有利于日后对自媒体的管理; 而对于自媒体传播的负面消息, 除 尽量满足受众对事实的需要, 也要将其传播影响规范在一个可控的范围内, 对于其后期造成 的不良影响, 也要积极地进行消除。

\section{7. 结语}

自媒体发展在我国方兴未艾，但我国对自媒体的管理和规范还并没有形成一个良好的机 制, 自媒体传播者也呈现出良莠不齐的情况, 自媒体的传播乱像正日益暴露在公众和受众的 眼里, 也对公众和受众造成极大的伤害。自媒体规范在接下来的一段时间, 都需要自媒体传 播者、政府、以及相关自媒体平台共同合作，为打造一个良好的自媒体传播氛围，减少自媒 体传播乱像，让自媒体走上规范的正途，是当前亟待解决的问题。

\section{致谢}

本文为江西师范大学新闻与传播学院教学建设专题项目《基于微信公众号的大学生思想政治 教育工作研究》的阶段性成果之一。

\section{References}

[1] Liu Kang. Video from the media status, characteristics and development trend of [J]. new media research, 2016,2 (20): 5-7+12.

[2] Wen Chao. The characteristics of information dissemination from the media and its impact on events: Taking the case of the failed vaccine in Shandong as an example, [J]. China Radio, 2016 (06): 33-36.

[3] Miao Lin. analysis of the characteristics and advantages and disadvantages of media communication from [J]., science and technology communication, 2016,8 (10): 39-40.

[4] Zhang Hongzhong, Liang Shuang, Zhang Shi Yu. The status quo and future of media development, [J]. news and writing, 2016 (05): 28-31.

[5] Guo Chen. Research on coping strategies of local governments in Internet public opinion [D]. Hainan University, 2016.

[6] Wang Xianbin. Research on China's government's online public opinion coping strategies under the new situation [D]. Shandong University, 2015.

[7] Liu Hongyu. Research on corporate public opinion in the new media environment [D]. Shaanxi Normal University, 2014.

[8] Shen Jinxia. Analysis of the characteristics of information dissemination from the media [J]. today media, 2012,20 (09): 94-96. 